\title{
Canopy Gap Colonization in the Atlantic Montane Rain Forest
}

\author{
Renato A. Ferreira de Lima* and Leila Cunha de Moura \\ Departamento de Ecologia; Universidade Estadual Paulista; Campus Rio Claro; Av. 24A, n. 1515; \\ raflima@esalq.usp.br; 13506-900; C.P. 199.; Rio Claro - SP - Brasil
}

\begin{abstract}
In the Atlantic Montane Rain Forest of South-eastern Brazil, a study was carried out to describe and evaluate canopy gap colonization. Gap composition by herb species was assessed through their soil coverage and woody species by measuring and identifying all individuals taller than one meter. Gap structure (gap size, number and diameter of treefalls), topographic position and surrounding vegetation were also measured. Two genera of Marantaceae were markedly frequent and abundant inside gaps. The more abundant and frequent woody species were shade tolerant. Species-rich families found inside gaps did not differ from the forest as a whole. Results revealed that direct and indirect influences of topography were important to determine gap composition of woody species. Evidently gap colonization had a considerable influence of topography and pre-established individuals besides variables of gap structure.
\end{abstract}

Key words: Gap floristics, south-eastern Brazil, marantaceae species, montane rain forest, topography

\section{INTRODUCTION}

In tropical and temperate forests, canopy gaps are regarded as processes of great importance to forest regeneration (Hartshorn, 1978; Denslow, 1980; Brokaw and Busing, 2000; Schnitzer and Carson, 2001). Gap environments influence plant populations in their growth (Garwood, 1983; Brown, 1993), herbivory (Coley, 1983), fruit production (Collins et al., 1985) and seed dispersal (Levey, 1988). Even physically, treefall events can damage plant individuals, sometimes being lethal (Clark and Clark, 1989).

However, the relation between gaps and species distribution in the forest is not so clear. Recent studies found that chance and other factors controlling the composition of the pool of species would be equally or more important to determinate species distribution than gap environment itself (Hubbell et al., 1999; Brokaw and Busing, 2000). Gap area not always is useful to explain gap partitioning (Dalling et al., 1998), and except in cases of large disturbance magnitude, the previous context of soil seed bank and pre-established individuals strongly determined gap composition (Garwood, 1983; Raich and Christensen, 1989).

Another important issue is the influence of canopy gaps in the species composition of forest communities. In tropical forests, an average gap would be formed by a single or double treefall event (Chandrashekara and Ramakrishnan, 1994), creating a canopy opening area ranging from 46 to $85 \mathrm{~m}^{2}$ (Brokaw, 1982; Sanford Jr. et al., 1986; Jans et al., 1993). Thus, as microclimate contrast varies according to the opening size (Barton et al., 1989), the average gap microclimate in tropical forests

${ }^{*}$ Author for correspondence 
does not differ sufficiently from closed canopy environments to stimulate light dependent species (Sanford Jr. et al., 1986; Brown, 1993). In this context, investigators have claimed that the colonization of gaps via pre-established individuals would be a prominent regeneration strategy (Garwood, 1983; Whitmore, 1989).

Considering the small number of studies developed in the Atlantic Montane Rain Forest, the main purpose of this study was to characterize the colonization of canopy gaps at this forest formation. This study also aimed to understand the factors constraining gap colonization of herb and woody species, how composition and abundance of species were influenced by variables of gap structure (gap area, and number and diameter of treefalls), by topography and by the gap sourrounding vegetation.

\section{MATERIAL AND METHODS}

This study was conducted in the Carlos Botelho State Park (PECB: $24^{\circ} 20^{\prime} \mathrm{S}, 47^{\circ} 44^{\prime} \mathrm{W}$ ), a 37.645 ha reserve located in the São Paulo State Atlantic Rain Forest, South-Eastern Brazil. Since 1993, the park is part of the Mata Atlântica Biosphere Reserve - UNESCO, and, in 1999, the same institution inscribed the park area within the Atlantic Forest South-East Reserves (World Natural Heritage Site). According to Domingues et al. (1993), the average annual rainfall is $1683 \mathrm{~mm}$ and the mean monthly temperature ranges from 14.5 to $22.4^{\circ} \mathrm{C}$. Local altitude varies from 700 to $900 \mathrm{~m}$. The study site used to conduct gap survey was located in an old growth forest covering approximately 150 ha. Local vegetation has been classified as Tropical Montane Rain Forest, with canopy height varying from 20 to $35 \mathrm{~m}$. In the understorey, the palm-heart (Euterpe edulis Mart.), species of the genera Geonoma and Psychotria, and tree ferns (Cyatheaceae) are easily found. There was no recent evidence of human disturbance in the study area.

During gap survey in 2002, 24 canopy gaps were selected and measured. This selection was based on the successional phase of gaps so that advanced phases of regeneration were not considered. Gaps in late successional phase were identified based on advanced decay of treefall debris, on the advanced height of the regenerating vegetation (> 10m: Gale, 2000) and on the aspect of the revolved soil in case of uprooted trees (Jans et al., 1993; Poorter et al., 1994). In the vegetation analysis gaps that clearly failed to regenerate as a result of a massive invasion of lianas or woody bamboos were excluded.

The definition used to delimitate gaps and to estimate their areas followed Brokaw (1982) and Lima (2005), respectively. For each gap, the topographic position (valley, slope and ridge) and the number and diameter at breast height (DBH) of the treefalls involved in the gap event were noted. Some stand measures from gap surrounding vegetation were also made. All canopy trees used to delimit the gap perimeter (sensu Brokaw 1982) had their DBH, trunk height and total height estimated. Estimates of tree height were made visually, always by the same observer and with the help of a scale of known length.

Inside each gap, the herb layer was measured using the line intercept method (Mueller-Dombois and Ellemberg, 1974), using eight radial oriented transects (N, NE, E, SE, etc.) with equal distance between them $\left(45^{\circ}\right)$. By this means, the colonization of the herb stratum inside gaps was evaluated based on the proportion of soil coverage, obtained by dividing the length of each group by the total length of transects. Treefall debris (dead trunks and branches) and woody bamboos were also measured, and were evaluated using the same line intercept method as for the herbs. The herb stratum was divided in the following groups: Calathea spp. (Marantaceae), Ctenanthe spp. (Marantaceae), herb ferns (mainly Didymoclaena truncatula (Sw.) J. Sm., Lastreopsis amplissima (C. Presl) Tindale and Stigmatopteris heterocarpa Rosenst.), ground bromeliads, woody bamboos, herbaceous bamboos (i.e. Chusquea spp., Olyra spp.). These groups represented the most abundant species on the herb stratum in the study site. Other two groups to be considered were the uncovered soil and other components such as low frequency herbs and woody seedlings.

The woody community was assessed including only plants taller than one meter and excluding life forms woody lianas and woody ferns. Individuals were identified to the level of species. Some herb species taller than one meter were also included in the analysis. The presence of broken stems and consequent sprouting was also noted. The classification of species into ecological groups was made based on other works (Klein, 1980; Tabarelli, 1997; Gandolfi, 2000) and on field observation in the study area. The variability in 
species relative abundance related to topography was evaluated by the non-parametrical Friedman ANOVA, after correcting the abundance of species in relation to the sampled area of each topographic position.

Table 1 - Proportions of soil coverage for each group of the herb stratum calculated in 24 canopy gaps. In parentheses, values obtained using only the gaps where the group soil coverage were other than zero.

\begin{tabular}{lrccc}
\multicolumn{1}{c}{ Group } & Average \pm SE & Frequency & \multicolumn{1}{c}{ Range } \\
\hline Calathea spp. & $4.44(5.85) \pm 3.57(3.88)$ & 70.83 & $(1.91)-73.92$ \\
Ctenanthe spp. & $6.04(6.72) \pm 1.41(1.37)$ & 87.50 & $(0.37)-24.63$ \\
Herb ferns & $3.43(6.31) \pm 1.28(1.25)$ & 83.33 & $(0.57)-21.01$ \\
Bromeliads & $1.03(1.23) \pm 0.32(0.33)$ & 87.50 & $(0.28)-7.62$ \\
Woody bamboo & $(1.27) \pm 0.38(0.63)$ & 25.00 & $(0.29)-7.77$ \\
Herb bamboo & $1.78(2.70) \pm 0.81(0.83)$ & 83.33 & $(0.30)-15.32$ \\
Uncovered soil & $39.46 \pm 1.95$ & 100.00 & $18.38-55.59$ \\
Other components & $15.98 \pm 1.46$ & 100.00 & $2.70-30.08$ \\
Treefall debris & $13.33 \pm 1.99$ & 100.00 & $1.26-35.42$ \\
\hline
\end{tabular}

\section{RESULTS}

The 24 canopy gaps evaluated in this study had values of area ranging from 15.15 to $276.47 \mathrm{~m}^{2}$ and total gap area sampled equal to $2060 \mathrm{~m}^{2}$. Values of soil coverage are shown in Table 1. Gap colonization from the herb stratum varied greatly, but some tendencies could be noted. Calathea showed higher values of soil coverage in valley gaps, and Ctenanthe was more abundant in ridge gaps. The Spearman correlation coefficient applied to these values of soil coverage (after transforming the proportions by arcsine $\sqrt{\mathrm{x}}$ ), revealed that these two genera had considerable negative correlation $\left(r_{s}=-0.710, N=24, p<0.0002\right)$. Even other less abundant groups, such as herb ferns and herb bamboos, were more correlated to topographic positioning than to any other gap characteristic (i.e. gap area), tending to be more abundant in ridges (respectively: $r_{s}=0.493, N=24, p<0.02 ; r_{s}=$ $0.438, \mathrm{~N}=24, \mathrm{p}<0.04)$.

Table 2 - Descriptive summary of the stand characteristics surrounding the 24 canopy gaps evaluated

\begin{tabular}{l|c|c}
\multicolumn{1}{c}{$\begin{array}{c}\text { Stand parameter } \\
(\mathbf{m})\end{array}$} & \multicolumn{2}{c}{ Sampled trees $(\mathbf{N}=\mathbf{3 3 7})$} \\
\cline { 2 - 3 } & Mean $\pm \mathbf{S E}$ & \multicolumn{1}{c}{ Range } \\
\hline Crown height & $4.69 \pm 0.09$ & $1.50-10.00$ \\
Stem height & $8.57 \pm 0.16$ & $3.00-18.00$ \\
Total tree height & $13.24 \pm 0.18$ & $7.00-24.00$ \\
Diameter & $0.36 \pm 0.01$ & $0.10-1.05$ \\
\hline
\end{tabular}

Another noteworthy result was related to the percentage of uncovered soil, which presented the highest values of overall average soil species coverage. This parameter presented a negative correlation with the number of falls $\left(\mathrm{r}_{\mathrm{s}}=-0.46, \mathrm{~N}=\right.$ $24, \mathrm{p}<0.03$ ), which suggested event intensity (increase of fallen wood mass). The uncovered soil percentage also presented negative correlation with the soil coverage of Calathea $\left(\mathrm{r}_{\mathrm{s}}=-0.43, \mathrm{~N}=\right.$ 24, $\mathrm{p}<0.04)$. This Marantaceae genus, with big leaves and fast growth habits (reaching $73.9 \%$ of soil coverage inside gaps), seemed to influence space availability within gaps. As expected, the soil coverage of treefall debris showed high frequency and positive correlations with gap size $\left(\mathrm{r}_{\mathrm{s}}=0.69, \mathrm{~N}=24, \mathrm{p}<0.0003\right)$ and number of treefall $\left(\mathrm{r}_{\mathrm{s}}=0.62, \mathrm{~N}=24, \mathrm{p}<0.002\right)$.

The results obtained for surrounding gap vegetation (see methods for details) are summarized in Table 2. Crown height presented a 
strong correlation to total tree height $\left(\mathrm{r}_{\mathrm{s}}=0.578, \mathrm{P}<\right.$ 0.001 ) and only a weak correlation with trunk height $\left(r_{s}=0.151, P=0.012\right)$, suggesting a variety of crown architecture around gaps. Indeed, wider trees were related to larger heights $\left(\mathrm{r}_{\mathrm{s}}=0.546, \mathrm{P}<\right.$ $0.001)$ and larger crowns $\left(r_{s}=0.556, P<0.001\right)$. No significant correlation was found between any stand parameter and gap area. The Kruskal-Wallis test was performed to analyze differences in the measured stand parameters across hills. As a result, the mean crown and total height of surrounding gap trees were both different between all topographic positions (respectively: $\mathrm{H}=9.94$, $\mathrm{P}=0.007$ and $\mathrm{H}=5.81, \mathrm{P}=0.055$ ), while mean wood volume was only different significantly between ridges and slopes $(\mathrm{H}=3.44, \mathrm{P}=0.06)$. Therefore, slopes tended to present stands with taller trees with broader crowns, followed by valley and ridge stands.

Considering only the individuals higher than $1 \mathrm{~m}$, individuals sampled belonged to 47 botanic families and 168 species. Families with higher abundance were Myrtaceae (16.43\%), Lauraceae, Palmae (12.78\%), Rubiaceae (9.52\%) and Leguminosae $(9.26 \%)$, while the families with greater species richness were Myrtaceae (32), Lauraceae (19), Leguminosae (15), Melastomataceae (14) and Rubiaceae (12). The most abundant species found were also the most frequent ones. In relation to the ecological groups of species, $75.0 \%$ were classified as shadetolerant, $10.1 \%$ as secondary, and $4.2 \%$ as pioneer species (no data was found for the remaining $10.7 \%)$.

Table 3 - Species found in Atlantic Montane Rain Forest canopy gaps and the respective abundance, frequency and ecological classification $(\mathrm{M}=$ shade tolerant, $\mathrm{S}=$ secondary and $\mathrm{O}=$ pioneer species; $\mathrm{ND}=$ no data available) . Results of Friedman ANOVAs for topography differences among species relative abundances are presented only for the most abundant species and when differences were significant $(P<0.05)$; topographic positions which differ (Multiple Comparison Test; $P<0.05$ ) are indicated by superscript different letters.

\begin{tabular}{|c|c|c|c|c|c|c|}
\hline \multirow{2}{*}{ Species } & \multirow{2}{*}{ Abundance } & \multirow{2}{*}{ Frequency } & \multirow{2}{*}{$\begin{array}{l}\text { Ecol. } \\
\text { group }\end{array}$} & \multicolumn{3}{|c|}{ Topographic position (\%) } \\
\hline & & & & Valley & Slope & Ridge \\
\hline Euterpe edulis Mart. & $52(6.78 \%)$ & $20(83.33 \%)$ & M & $35.41^{\mathrm{a}}$ & $21.14^{\mathrm{b}}$ & $43.45^{\mathrm{a}}$ \\
\hline Bathysa australis Hook. & $28(3.65 \%)$ & $10(41.67 \%)$ & $\mathrm{S}$ & $62.16^{\mathrm{a}}$ & $31.88^{\mathrm{b}}$ & $5.96^{\mathrm{b}}$ \\
\hline Ardisia guianensis (Aublet.) Mez & $21(2.74 \%)$ & $11(45.83 \%)$ & M & $45.20^{\mathrm{a}}$ & $54.80^{\mathrm{a}}$ & $-b$ \\
\hline Geonoma gamiova Rodr. & $21(2.74 \%)$ & $9(37.50 \%)$ & M & $54.92^{\mathrm{a}}$ & $45.08^{\mathrm{a}}$ & $-\mathrm{b}$ \\
\hline Pouteria bullata (S. Moore) Baehni & $16(2.09 \%)$ & $11(45.83 \%)$ & $\mathrm{M}$ & 24.83 & 37.07 & 38.10 \\
\hline Inga sessilis (Vell.) Mart. & $15(1.96 \%)$ & $7(29.17 \%)$ & S & $41.50^{\mathrm{a}}$ & $22.13^{\mathrm{b}}$ & $36.37^{\mathrm{a}}$ \\
\hline $\begin{array}{l}\text { Sorocea bomplandii (Baill.) Burger, } \\
\text { Lanjow and Boer }\end{array}$ & $15(1.96 \%)$ & $6(25.00 \%)$ & M & 15.07 & 61.83 & 23.10 \\
\hline Calyptranthes lanceolata Berg. & $14(1.83 \%)$ & $9(37.50 \%)$ & M & 39.99 & 47.74 & 12.27 \\
\hline Endlicheria paniculata (Spreng.) Macbr. & $13(1.69 \%)$ & $7(29.17 \%)$ & M & 34.77 & 51.90 & 13.33 \\
\hline $\begin{array}{l}\text { Rapanea hermogenesii Jung. Mendaçolli } \\
\text { and Bernacci }\end{array}$ & $13(1.69 \%)$ & $9(37.50 \%)$ & M & $22.04^{\mathrm{a}}$ & $32.90^{\mathrm{b}}$ & $45.07^{\mathrm{b}}$ \\
\hline Ocotea brachybotra (Meissn.) Mez & $13(1.69 \%)$ & $10(41.67 \%)$ & M & $13.39^{\mathrm{a}}$ & $24.99^{\mathrm{a}}$ & $61.62^{\mathrm{b}}$ \\
\hline Ocotea catharinensis $\mathrm{Mez}$ & $13(1.69 \%)$ & $10(41.67 \%)$ & M & 20.83 & 25.91 & 53.26 \\
\hline Cupania oblongifolia Cambess. & $12(1.56 \%)$ & $6(25.00 \%)$ & M & $60.77^{\mathrm{a}}$ & $25.91^{\mathrm{b}}$ & $13.32^{\mathrm{b}}$ \\
\hline Dichorisandra pubescens Mart. & $12(1.56 \%)$ & $7(29.17 \%)$ & M & 16.91 & 44.17 & 38.91 \\
\hline Esembeckia grandiflora Mart. & $12(1.56 \%)$ & $6(25.00 \%)$ & M & $-^{\mathrm{a}}$ & $8.87^{\mathrm{b}}$ & $91.13^{\mathrm{c}}$ \\
\hline
\end{tabular}




\begin{tabular}{|c|c|c|c|c|c|c|}
\hline Matayba juglandiflora (Cambess.) Radlk. & $12(1.56 \%)$ & $6(25.00 \%)$ & M & $14.93^{\mathrm{a}}$ & $27.84^{\mathrm{b}}$ & $57.23^{\mathrm{c}}$ \\
\hline Andira anthelmia (Vog.) Benth. & $11(1.43 \%)$ & $8(33.33 \%)$ & M & - & 36.86 & 63.14 \\
\hline Cryptocarya moschata Nees and Mart. & $11(1.43 \%)$ & $7(29.17 \%)$ & M & 61.66 & 38.34 & - \\
\hline Eugenia stictosepala Kiaersk. & $11(1.43 \%)$ & $7(29.17 \%)$ & M & $7.16^{\mathrm{a}}$ & $16.02^{\mathrm{b}}$ & $76.82^{\mathrm{c}}$ \\
\hline Mollinedia schottiana (Spreng.) Perk. & $10(1.30 \%)$ & $9(37.50 \%)$ & M & $24.67^{\mathrm{a}}$ & $12.27^{\mathrm{a}}$ & $63.06^{\mathrm{b}}$ \\
\hline Ocotea teleiandra (Meissn.) Mez & $10(1.30 \%)$ & $5(20.83 \%)$ & M & $22.84^{\mathrm{a}}$ & $59.65^{\mathrm{b}}$ & $17.51^{\mathrm{a}}$ \\
\hline Posoqueria acutifolia Mart. & $10(1.30 \%)$ & $6(25.00 \%)$ & M & $9.92^{\mathrm{a}}$ & $44.42^{\mathrm{b}}$ & $45.66^{\mathrm{b}}$ \\
\hline Alibertia myrcifolia K. Schum. & $9(1.17 \%)$ & $8(33.33 \%)$ & M & - & 55.56 & 44.44 \\
\hline Cordia sellowiana Cham. & $9(1.17 \%)$ & $4(16.67 \%)$ & S & - & 63.00 & 37.00 \\
\hline Myrcia fallax (Rich.) DC. & $9(1.17 \%)$ & $5(20.83 \%)$ & M & 9.17 & 20.52 & 70.31 \\
\hline Myrcia pubipetala Miq. & $9(1.17 \%)$ & $7(29.17 \%)$ & M & 9.88 & 29.49 & 60.62 \\
\hline Ocotea dispersa (Nees) Mez & $9(1.17 \%)$ & $7(29.17 \%)$ & M & 30.49 & 22.75 & 46.76 \\
\hline Eugenia cerasiflora Miq. & $8(1.04 \%)$ & $4(16.67 \%)$ & M & 62.30 & 18.59 & 19.11 \\
\hline $\begin{array}{l}\text { Myrceugenia myrcioides (Cambess.) } \\
\text { Berg. }\end{array}$ & $8(1.04 \%)$ & $4(16.67 \%)$ & M & 56.73 & 8.47 & 34.80 \\
\hline Piper cf. chimonanthifolium Kunth & $8(1.04 \%)$ & $5(20.83 \%)$ & ND & 57.27 & 42.73 & - \\
\hline Copaifera langsdorfii Desf. & $7(0.91 \%)$ & $6(25.00 \%)$ & M & - & 7.50 & 92.50 \\
\hline Copaifera trapezifolia Hayne & $7(0.91 \%)$ & $5(20.83 \%)$ & M & - & 54.88 & 45.12 \\
\hline Leandra mosenii Cogn. & $7(0.91 \%)$ & $2(8.33 \%)$ & $S$ & 18.26 & 81.74 & - \\
\hline Miconia pusilliflora Triana & $7(0.91 \%)$ & $6(25.00 \%)$ & M & - & 26.73 & 73.27 \\
\hline Piper caldense C. DC. & $7(0.91 \%)$ & $4(16.67 \%)$ & M & 88.94 & 11.06 & - \\
\hline Plinia complanata Kawasaki and Holst. & $7(0.91 \%)$ & $4(16.67 \%)$ & M & 18.26 & 81.74 & - \\
\hline $\begin{array}{l}\text { Pouteria caimito (Ruiz and Pávora) } \\
\text { Radlk. }\end{array}$ & $7(0.91 \%)$ & $2(8.33 \%)$ & M & - & 26.73 & 73.27 \\
\hline Rudgea jasminioides (Cham.) Müll. Arg. & $6(0.78 \%)$ & $5(20.83 \%)$ & M & 77.01 & 22.99 & - \\
\hline Cabralea canjerana (Vell.) Mart. & $6(0.78 \%)$ & $5(20.83 \%)$ & M & 27.22 & 10.16 & 62.62 \\
\hline $\begin{array}{l}\text { Marlierea eugenipsoides (Legr. and } \\
\text { Kaus.) Legr. }\end{array}$ & $6(0.78 \%)$ & $4(16.67 \%)$ & ND & - & 32.73 & 67.27 \\
\hline Miconia doriana Cogn. & $6(0.78 \%)$ & $1(4.17 \%)$ & $\mathrm{O}$ & - & 100.00 & - \\
\hline Myrcia sp. & $6(0.78 \%)$ & $3(12.50 \%)$ & M & 87.02 & 12.98 & - \\
\hline Ocotea daphnifolia (Meisn.) Mez & $6(0.78 \%)$ & $4(16.67 \%)$ & M & - & 19.57 & 80.43 \\
\hline Piper pseudopothifolium C. DC. & $6(0.78 \%)$ & $5(20.83 \%)$ & ND & 30.49 & 22.75 & 46.76 \\
\hline $\begin{array}{l}\text { Alchornea triplinervia (Spreng.) Müll. } \\
\text { Arg. }\end{array}$ & $5(0.65 \%)$ & $3(12.50 \%)$ & M & 47.19 & 52.81 & - \\
\hline
\end{tabular}


Cont. Table 3

\begin{tabular}{|c|c|c|c|c|c|c|}
\hline Casearia decandra Jacq. & $5(0.65 \%)$ & $5(20.83 \%)$ & M & 25.10 & 74.90 & - \\
\hline Geonoma schottiana Mart. & $5(0.65 \%)$ & $3(12.50 \%)$ & M & - & - & 100.00 \\
\hline Inga sellowiana Benth. & $5(0.65 \%)$ & $5(20.83 \%)$ & M & 15.75 & 11.76 & 72.49 \\
\hline Marlierea obscura Legr. & $5(0.65 \%)$ & $4(16.67 \%)$ & M & 56.82 & 14.13 & 29.05 \\
\hline Sclerolobium denudatum Vog. & $5(0.65 \%)$ & $4(16.67 \%)$ & M & - & 24.49 & 75.51 \\
\hline Zollernia illicifolia Vog. & $5(0.65 \%)$ & $3(12.50 \%)$ & M & 15.75 & 11.76 & 72.49 \\
\hline Allophylus petiolulatus Radlk. & $4(0.52 \%)$ & $2(8.33 \%)$ & M & - & 100.00 & - \\
\hline Amaioua intermedia Mart. & $4(0.52 \%)$ & $4(16.67 \%)$ & M & - & 13.95 & 86.05 \\
\hline Calycorectes australis Legr. & $4(0.52 \%)$ & $3(12.50 \%)$ & M & 30.88 & 69.12 & - \\
\hline Chionanthus filiformis (Vell.) P.S. Grenn & $4(0.52 \%)$ & $3(12.50 \%)$ & M & - & 32.73 & 67.27 \\
\hline $\begin{array}{l}\text { Chomelia catharinae (L.B. Sm. and } \\
\text { Downs) Steyerm. }\end{array}$ & $4(0.52 \%)$ & $3(12.50 \%)$ & M & - & 32.73 & 67.27 \\
\hline Eugenia neoglomerata Sobr. & $4(0.52 \%)$ & $2(8.33 \%)$ & M & - & 100.00 & - \\
\hline Eugenia subavenia Berg. & $4(0.52 \%)$ & $4(16.67 \%)$ & M & 20.77 & 15.50 & 63.72 \\
\hline Guapira opposita (Vell.) Reitz & $4(0.52 \%)$ & $4(16.67 \%)$ & M & 24.84 & 37.07 & 38.10 \\
\hline Hyeronima alchorneoides Fr. All. & $4(0.52 \%)$ & $3(12.50 \%)$ & M & 24.84 & 37.07 & 38.10 \\
\hline Jacaranda cf. montana Morav. & $4(0.52 \%)$ & $4(16.67 \%)$ & S & - & 100.00 & - \\
\hline Miconia sellowiana Naud. & $4(0.52 \%)$ & $2(8.33 \%)$ & $\mathrm{O}$ & - & 32.73 & 67.27 \\
\hline Myrceugenia sp. & $4(0.52 \%)$ & $3(12.50 \%)$ & M & - & 59.34 & 40.66 \\
\hline Myrocarpus frondosus Fr. All. & $4(0.52 \%)$ & $4(16.67 \%)$ & M & - & 32.73 & 67.27 \\
\hline Rapanea gardneriana (A. DC.) Mez & $4(0.52 \%)$ & $3(12.50 \%)$ & $S$ & - & 59.34 & 40.66 \\
\hline Ocotea silvestris Vatt. & $4(0.52 \%)$ & $4(16.67 \%)$ & M & 24.84 & 37.07 & 38.10 \\
\hline Parinari excelsa Sabine & $4(0.52 \%)$ & $4(16.67 \%)$ & M & - & 13.95 & 86.05 \\
\hline Rubiaceae & $4(0.52 \%)$ & $3(12.50 \%)$ & ND & - & 32.73 & 67.27 \\
\hline Tabebuia serratifolia (Vahl.) Nich. & $4(0.52 \%)$ & $3(12.50 \%)$ & M & - & 32.73 & 67.27 \\
\hline Bactris cf. setosa Mart. & $3(0.39 \%)$ & $3(12.50 \%)$ & M & 72.83 & 27.17 & - \\
\hline Byrsonima ligustrifolia Juss. & $3(0.39 \%)$ & $3(12.50 \%)$ & M & - & 49.32 & 50.68 \\
\hline Dalbergia frutescens (Vell.) Britton & $3(0.39 \%)$ & $3(12.50 \%)$ & S & - & 19.57 & 80.43 \\
\hline Guatteria australis A.St. -Hil. & $3(0.39 \%)$ & $3(12.50 \%)$ & M & - & 49.32 & 50.68 \\
\hline Matayba guianensis Aubl. & $3(0.39 \%)$ & $2(8.33 \%)$ & M & 40.12 & 59.88 & - \\
\hline Miconia cabussu Hoehne & $3(0.39 \%)$ & $3(12.50 \%)$ & S & 56.60 & - & 43.40 \\
\hline Leandra sp. & $3(0.39 \%)$ & $1(4.17 \%)$ & ND & - & 100.00 & - \\
\hline
\end{tabular}


Cont. Table 3

\begin{tabular}{|c|c|c|c|c|c|c|}
\hline Neomitranthes obscura (DC.) Legr. & $3(0.39 \%)$ & $3(12.50 \%)$ & M & 30.49 & 22.75 & 46.76 \\
\hline Ocotea bicolor Vatt. & $3(0.39 \%)$ & $3(12.50 \%)$ & M & 56.60 & - & 43.40 \\
\hline Piptocarpha axillaries (Less.) Backer & $3(0.39 \%)$ & $2(8.33 \%)$ & $\mathrm{O}$ & - & 19.57 & 80.43 \\
\hline Psychotria forsteronioides Müll. Arg. & $3(0.39 \%)$ & $2(8.33 \%)$ & M & - & 100.00 & - \\
\hline Pterocarpus rohrii Vahl. & $3(0.39 \%)$ & $3(12.50 \%)$ & M & - & 49.32 & 50.68 \\
\hline Tetrorchidium rubrivenium Poepp. & $3(0.39 \%)$ & $1(4.17 \%)$ & $S$ & 100.00 & - & - \\
\hline $\begin{array}{l}\text { Xylopia langsdorfiana St. Hilaire and } \\
\text { Tulasne }\end{array}$ & $3(0.39 \%)$ & $3(12.50 \%)$ & M & - & 19.57 & 80.43 \\
\hline Aspidosperma olivaceum Müll. Arg. & $2(0.26 \%)$ & $2(8.33 \%)$ & M & - & 32.73 & 67.27 \\
\hline Calyptrahthes aff. lucida Mart. ex DC. & $2(0.26 \%)$ & $2(8.33 \%)$ & M & - & 32.73 & 67.27 \\
\hline Capsicum recurbatum Sendtn. & $2(0.26 \%)$ & $2(8.33 \%)$ & $\mathrm{O}$ & - & 100.00 & - \\
\hline Cecropia glaziouii Snethl. & $2(0.26 \%)$ & $1(4.17 \%)$ & $\mathrm{O}$ & 100.00 & - & - \\
\hline $\begin{array}{l}\text { Cinamomum triplinerve (Ruiz and Pavón) } \\
\text { Kosterm }\end{array}$ & $2(0.26 \%)$ & $1(4.17 \%)$ & M & - & 100.00 & - \\
\hline Cryptocarya aschersoniana Mez. & $2(0.26 \%)$ & $2(8.33 \%)$ & M & 39.47 & - & 60.53 \\
\hline Dalbergia foliolosa Benth. & $2(0.26 \%)$ & $2(8.33 \%)$ & S & 57.27 & 42.73 & - \\
\hline Drymis winterii Forst. & $2(0.26 \%)$ & $2(8.33 \%)$ & M & - & 32.73 & 67.27 \\
\hline Eugenia beaurepaireana (Kiaersk.) Legr. & $2(0.26 \%)$ & $1(4.17 \%)$ & M & - & - & 100.00 \\
\hline Eugenia cereja Legr. & $2(0.26 \%)$ & $2(8.33 \%)$ & M & - & 100.00 & - \\
\hline Eugenia sp. 1 & $2(0.26 \%)$ & $1(4.17 \%)$ & ND & 100.00 & - & - \\
\hline Eugenia sp. 3 & $2(0.26 \%)$ & $1(4.17 \%)$ & ND & - & 100.00 & - \\
\hline Hirtella hebeclada Moric. ex A. DC. & $2(0.26 \%)$ & $2(8.33 \%)$ & M & - & 32.73 & 67.27 \\
\hline Ixora burchelliana Müll. Arg. & $2(0.26 \%)$ & $2(8.33 \%)$ & M & - & - & 100.00 \\
\hline Marlierea parviflora $\mathrm{O}$. Berg. & $2(0.26 \%)$ & $2(8.33 \%)$ & M & 100.00 & - & - \\
\hline Maytenus robusta Reiss. & $2(0.26 \%)$ & $2(8.33 \%)$ & M & - & 32.73 & 67.27 \\
\hline Miconia petropolitana Cogn. & $2(0.26 \%)$ & $1(4.17 \%)$ & M & - & - & 100.00 \\
\hline Miconia racemifera (DC.) Triana & $2(0.26 \%)$ & $2(8.33 \%)$ & ND & - & - & 100.00 \\
\hline Ocotea aciphylla (Nees) Mez & $2(0.26 \%)$ & $2(8.33 \%)$ & M & - & - & 100.00 \\
\hline Ocotea glaziovii Mez & $2(0.26 \%)$ & $2(8.33 \%)$ & M & - & - & 100.00 \\
\hline Ocotea pulchra Vatt. & $2(0.26 \%)$ & $1(4.17 \%)$ & M & - & 100.00 & - \\
\hline Persea pyrifolia Nees and Mart. ex Nees & $2(0.26 \%)$ & $2(8.33 \%)$ & M & 39.47 & - & 60.53 \\
\hline Plinia pauciflora Kawasaki and Holst. & $2(0.26 \%)$ & $2(8.33 \%)$ & M & - & 32.73 & 67.27 \\
\hline Protium heptaphillum (Aublet.) March. & $2(0.26 \%)$ & $2(8.33 \%)$ & M & - & 32.73 & 67.27 \\
\hline
\end{tabular}


Cont. Table 3

\begin{tabular}{|c|c|c|c|c|c|c|}
\hline Rollinia parviflora A.St. -Hil. & $2(0.26 \%)$ & $2(8.33 \%)$ & M & - & 32.73 & 67.27 \\
\hline Tapirira guianensis Aublet. & $2(0.26 \%)$ & $2(8.33 \%)$ & M & - & - & 100.00 \\
\hline Vantanea compacta (Schnizl.) Cuatr. & $2(0.26 \%)$ & $2(8.33 \%)$ & M & - & - & 100.00 \\
\hline Vernonia puberula Less. & $2(0.26 \%)$ & $2(8.33 \%)$ & M & 100.00 & - & - \\
\hline Acacia sp. & $1(0.13 \%)$ & $1(4.17 \%)$ & S & - & 100.00 & - \\
\hline Aniba viridis $\mathrm{Mez}$ & $1(0.13 \%)$ & $1(4.17 \%)$ & M & - & 100.00 & - \\
\hline Begonia capanemae Brade & $1(0.13 \%)$ & $1(4.17 \%)$ & M & - & 100.00 & - \\
\hline Buchenavia kleinii Exell. & $1(0.13 \%)$ & $1(4.17 \%)$ & M & - & 100.00 & - \\
\hline Casearia obliqua Sprengel & $1(0.13 \%)$ & $1(4.17 \%)$ & M & - & 100.00 & - \\
\hline Casearia sylvestris $\mathrm{Sw}$. & $1(0.13 \%)$ & $1(4.17 \%)$ & $S$ & 100.00 & - & - \\
\hline Citronella paniculata (Mart.) Howard & $1(0.13 \%)$ & $1(4.17 \%)$ & M & - & 100.00 & - \\
\hline Clethra scabra Pers. & $1(0.13 \%)$ & $1(4.17 \%)$ & $S$ & - & 100.00 & - \\
\hline Coccoloba martii Meissn. & $1(0.13 \%)$ & $1(4.17 \%)$ & M & - & 100.00 & - \\
\hline Costus spiralaris K. Schum. & $1(0.13 \%)$ & $1(4.17 \%)$ & M & 100.00 & - & - \\
\hline Coussapoa microcarpa (Schott) Rizz. & $1(0.13 \%)$ & $1(4.17 \%)$ & M & 100.00 & - & - \\
\hline Coussarea contracta (Walp.) Müll.Arg. & $1(0.13 \%)$ & $1(4.17 \%)$ & M & - & 100.00 & - \\
\hline Cupania vernalis Cambess & $1(0.13 \%)$ & $1(4.17 \%)$ & M & - & - & 100.00 \\
\hline Cybianthus peruvianus (A. DC.) Miq. & $1(0.13 \%)$ & $1(4.17 \%)$ & M & - & 100.00 & - \\
\hline Diploon cuspidatum (Hoenne) Cronquist & $1(0.13 \%)$ & $1(4.17 \%)$ & ND & - & 100.00 & - \\
\hline Ecclinusa ramiflora Mart. & $1(0.13 \%)$ & $1(4.17 \%)$ & M & 100.00 & - & - \\
\hline Erythroxylum ambiguum Peyr. & $1(0.13 \%)$ & $1(4.17 \%)$ & ND & - & 100.00 & - \\
\hline Eugenia capitulifera Berg. & $1(0.13 \%)$ & $1(4.17 \%)$ & M & - & 100.00 & - \\
\hline Eugenia cf. cambucarana Kiaersk. & $1(0.13 \%)$ & $1(4.17 \%)$ & M & - & 100.00 & - \\
\hline Eugenia cf. involucrata DC. & $1(0.13 \%)$ & $1(4.17 \%)$ & M & - & - & 100.00 \\
\hline Eugenia cf. umbelliflora Berg. & $1(0.13 \%)$ & $1(4.17 \%)$ & M & 100.00 & - & - \\
\hline Eugenia florida DC. & $1(0.13 \%)$ & $1(4.17 \%)$ & M & - & - & 100.00 \\
\hline Eugenia melanogina (D.Legrand) Sobral & $1(0.13 \%)$ & $1(4.17 \%)$ & ND & - & 100.00 & - \\
\hline Eugenia pruinosa Legr. & $1(0.13 \%)$ & $1(4.17 \%)$ & M & 100.00 & - & - \\
\hline Eugenia sp. 2 & $1(0.13 \%)$ & $1(4.17 \%)$ & ND & - & - & 100.00 \\
\hline $\begin{array}{l}\text { Garcinia gardneriana (Planch. and } \\
\text { Triana) D. Zappi }\end{array}$ & $1(0.13 \%)$ & $1(4.17 \%)$ & M & - & - & 100.00 \\
\hline Guarea macrophylla Vahl. & $1(0.13 \%)$ & $1(4.17 \%)$ & M & 100.00 & - & - \\
\hline
\end{tabular}


Cont. Table 3

\begin{tabular}{|c|c|c|c|c|c|c|}
\hline Ilex amara (Vell.) Loesener & $1(0.13 \%)$ & $1(4.17 \%)$ & M & - & 100.00 & - \\
\hline Inga capitata Desv. & $1(0.13 \%)$ & $1(4.17 \%)$ & M & 100.00 & - & - \\
\hline Lamanonia ternata Vell. & $1(0.13 \%)$ & $1(4.17 \%)$ & $\mathrm{M}$ & - & 100.00 & - \\
\hline Lauraceae & $1(0.13 \%)$ & $1(4.17 \%)$ & M & - & 100.00 & - \\
\hline Leandra pilonensis Wurdack & $1(0.13 \%)$ & $1(4.17 \%)$ & ND & - & - & 100.00 \\
\hline Leandra sabiaensis Brade & $1(0.13 \%)$ & $1(4.17 \%)$ & ND & - & 100.00 & - \\
\hline Picramnia cf. gardneri Planch. & $1(0.13 \%)$ & $1(4.17 \%)$ & ND & - & 100.00 & - \\
\hline Licania hoehnei Pilger & $1(0.13 \%)$ & $1(4.17 \%)$ & M & - & - & 100.00 \\
\hline Miconia cubatanensis Hoehne & $1(0.13 \%)$ & $1(4.17 \%)$ & $S$ & - & - & 100.00 \\
\hline Miconia theezans Cogn. & $1(0.13 \%)$ & $1(4.17 \%)$ & $\mathrm{S}$ & - & - & 100.00 \\
\hline Miconia valtherii Naudin & $1(0.13 \%)$ & $1(4.17 \%)$ & ND & - & 100.00 & - \\
\hline Mollinedia elegans Tul. & $1(0.13 \%)$ & $1(4.17 \%)$ & M & - & - & 100.00 \\
\hline Mollinedia oligantha Perk. & $1(0.13 \%)$ & $1(4.17 \%)$ & M & - & 100.00 & - \\
\hline $\begin{array}{l}\text { Myrceugenia glaucescens (Cambess.) } \\
\text { Legr. and Kausel }\end{array}$ & $1(0.13 \%)$ & $1(4.17 \%)$ & $\mathrm{M}$ & 100.00 & - & - \\
\hline Myrceugenia kleinii Legr. and Kaus & $1(0.13 \%)$ & $1(4.17 \%)$ & M & 100.00 & - & - \\
\hline Rapanea umbellata (Mart. ex DC.) Mez & $1(0.13 \%)$ & $1(4.17 \%)$ & M & - & 100.00 & - \\
\hline Nectandra oppositifolia Nees & $1(0.13 \%)$ & $1(4.17 \%)$ & M & - & 100.00 & - \\
\hline Ocotea elegans $\mathrm{Mez}$ & $1(0.13 \%)$ & $1(4.17 \%)$ & $\mathrm{M}$ & - & 100.00 & - \\
\hline Ormosia dasycarpa Jacks. & $1(0.13 \%)$ & $1(4.17 \%)$ & ND & - & 100.00 & - \\
\hline Piper aduncum L. & $1(0.13 \%)$ & $1(4.17 \%)$ & M & - & 100.00 & - \\
\hline Piptocarpha macropoda (DC.) Backer & $1(0.13 \%)$ & $1(4.17 \%)$ & $S$ & - & - & 100.00 \\
\hline Psychotria suterella Müll. Arg. & $1(0.13 \%)$ & $1(4.17 \%)$ & M & - & 100.00 & - \\
\hline Quina magalano-gomesii Schwacke & $1(0.13 \%)$ & $1(4.17 \%)$ & M & - & - & 100.00 \\
\hline Rollinia sericea R.E. Fries & $1(0.13 \%)$ & $1(4.17 \%)$ & $\mathrm{M}$ & - & 100.00 & - \\
\hline Simira sp. & $1(0.13 \%)$ & $1(4.17 \%)$ & ND & 100.00 & - & - \\
\hline Sloanea monosperma Vell. & $1(0.13 \%)$ & $1(4.17 \%)$ & $\mathrm{M}$ & 100.00 & - & - \\
\hline Solanum cinnamomeum Sendt. & $1(0.13 \%)$ & $1(4.17 \%)$ & $\mathrm{O}$ & - & 100.00 & - \\
\hline Solanum piluliferum Dunal & $1(0.13 \%)$ & $1(4.17 \%)$ & $\mathrm{O}$ & 100.00 & - & - \\
\hline Tibouchina pulchra Cogn. & $1(0.13 \%)$ & $1(4.17 \%)$ & S & 100.00 & - & - \\
\hline Weinmannia paulliniifolia Pohl. ex Ser. & $1(0.13 \%)$ & $1(4.17 \%)$ & M & 100.00 & - & - \\
\hline
\end{tabular}




\section{DISCUSSION}

It would be interesting to discuss the behavior of the soil coverage of the two Marantaceae genera (Calathea and Ctenanthe) in the study area, although the influence of herb layer on species regeneration was not directly evaluated in this study. With broad leaves and the habit of forming dense clusters, these herb components could produce thick layers of litter (Lima and Moura, personal observation) that strongly inhibited seed germination (Vázquez-Yanes et al., 1990). Within gaps, where herb species can enhance their seed emergence (Horvitz and Schemske, 1994) and growth (Collins et al., 1985; Chandrashekara and Ramakrishnan, 1994), intense herb coverage affects gap regeneration by woody species, either by depletion of available resources or by germination inhibition (Collins et al., 1985; George and Bazzaz, 1999).

These two genera were virtually self-excluding in relation to the topographic positioning. As at other forest sites (Basnet, 1992; Poorter et al., 1994; Oliveira-Filho et al., 1998; Gale, 2000), valleys are often more humid and dark than ridges that receive a more direct influence of wind and sunlight. The environmental contrast between valley and ridge sites also influences other herb groups such as herb ferns and herb bamboos. Thus, topography features seem to play an important role to herb species distribution inside gaps.

Although the relation between crown extension and tree height is influenced by species-to-species crown architecture, there was an average tendency of thicker crowns in higher trees around gaps $\left(\mathrm{r}_{\mathrm{s}}=\right.$ $0.615, \mathrm{P}<0.01, \mathrm{~N}=42$ ). It is known that daily gap insolation and direct light incidence are smaller in openings surrounded by trees with bigger crowns and total tree heights (Chazdon and Fetcher, 1984; Collins et al., 1985; Brown, 1993). Therefore, except for the time of the day when the sun is at high noon, light entrance, and thus microclimate (Collins et al., 1985), is different with respect to canopy height of the surrounding vegetation (Hubbell and Foster, 1986). Also, the results for surrounding gap vegetation revealed differences in stand parameters across hills. Ridges have smaller trees and lower diameters compared to other positions. It is likely that this source of variation reflects soil characteristics (Bellingham and Tanner, 2000) and certainly affects light entrance and disturbance regime in the forest (Gale, 2000; Robert and Moravie, 2003). Since these data were not conclusive, further studies must be carried out to test this possibility in the study area.

In spite of being present in virtually all gaps, the soil coverage of debris varied greatly. This parameter of gap structure was positively correlated with the number of falls and it acted directly on species establishment. The influence of fallen trunks and branches involves the mortality of pre-established individuals and the substrate conditions to seedling establishment (Hartshorn, 1978). Tabarelli (1997) found that soil coverage of dead material influences the stem density inside gaps in another Montane Rain Forest site near the study area (São Luís do Paraitinga, São Paulo, Brazil). Although stem mortality is a non-specific phenomenon, the influence of substrate conditions may vary between species (Brandani et al., 1988; Lawton and Putz, 1988; Núñez-Farfán and Dirzo, 1988). In the present study area, at least two species were noted as able to recruit over dead logs (Cecropia glaziouii and Leandra mosenii: authors' observation).

With respect to woody species, a study carried out in the same forest, Aguiar (2003) found very similar floristic results for the forest as a whole. Among the 9544 individuals sampled in the study, the families with higher abundance were Myrtaceae (14.59\%), Rubiaceae (12.58\%), Lauraceae (12.29\%), Palmae (11.38\%), and Leguminosae $(5.87 \%)$, and the more species-rich were Myrtaceae (58), Lauraceae (36), Leguminosae (17), Melastomataceae and Rubiaceae (12). The most abundant and speciesrich families were the same obtained in this study, suggesting little change of composition in canopy gaps.

As shown on Table 3, many species presented some degree of influence by the topographical position of gaps, being exclusive to one or two positions, or showing differences in their relative abundances across hills. This suggested that not only gap structure could determinate gap colonization. The environmental differences between valley and ridge sites, as long with other dispersal constraints (e.g., timing of dispersal, distance between the gap and the fruiting adults) could control the pool of species available for gap colonization (Bellingham and Tanner, 2000; Robert and Moravie, 2003). Otherwise, among the twenty most abundant species found in this study, only B. australis and I. sessilis did not correspond to the shade tolerant ecological group; many of them were typical understorey species (i.e. $G$. 
gamiova, Ardisia guianensis and Endlicheria paniculata: Table 3). Typically pioneer genera as Cecropia, Solanum, Leandra and some Miconia and Piper species had restricted occurrences (gap sizes $>100 \mathrm{~m}^{2}$ ) and low abundances.

In addition, $25.81 \%$ of all individuals presented broken stem and consequent sprouting, indicating individuals that survived after being hit in the fall event. Similar results were found at other forest sites (Uhl et al., 1988; Carvalho et al., 2000), where a considerable part of gap regeneration appeared to be happening through surviving individuals. This inference would enhance the suggestion that the environmental conditions previous to gap formation such as topography and the subsequent environmental changes (Carvalho et al., 2000), would play an important role in gap floristics via surviving individuals. This pattern of gap colonization would approximate the floristic of gaps and non-gap sites (Lieberman et al., 1989; Denslow and Hartshorn, 1994).

A similar result was found in montane rain forests in São Paulo State (Tabarelli and Mantovani, 2000) and in Minas Gerais State (Carvalho et al., 2000). Therefore, for Montane Rain Forests sites of South-eastern Brazil, evidence exists that the results found here would be a general pattern in gap colonization by woody species in this forest formation. The predominance of small gap sizes found in the study area would not create sufficient environment differences that could generate substantial floristic changes (Whitmore, 1989). Except in cases when there was soil exposure by uprooting (Lawton and Putz, 1988; VázquezYanes and Orozco-Segovia, 1993), regular forest gaps did not seem to create big changes on the community composition. In this context, only a few numbers of species had their distributions related to gap environments; the larger part of the individuals inside gaps were probably just benefited by chancy canopy openings, enhancing their growth rates (Lieberman et al., 1989; Schinitzer and Carson, 2001).

\section{ACKNOWLEDGEMENTS}

We would like to thank João B. Baitelo, Osny T. Aguiar, Sigrid Jung-Mendaçolli, Rosana Romero, João Renato Stehmann, Vinicius A. Dittrich and Vera L. Uliana for the identification of Lauraceae, Myrtaceae, Rubiaceae, Melastomataceae,
Pteridaceae and Marantaceae families, respectively. We are also thankful to Harold G. Fowler who gently reviewed this manuscript and to the Instituto Florestal de São Paulo for making this work possible.

\section{RESUMO}

Na Floresta Pluvial Atlântica Montana do Sudeste Brasileiro, foi realizado um estudo para descrever e avaliar a colonização de clareiras. A composição de clareiras foi levantada através da cobertura do solo para as espécies herbáceas enquanto que todos os indivíduos lenhosos maiores que um metro de altura foram mensurados e identificados. Também foram coletadas informações sobre a estrutura das clareiras (área da clareira, número e diâmetro das quedas), posição topográfica e vegetação circundante. Dois gêneros de Marantaceae apresentaram considerável freqüência e abundância nas clareiras. As espécies lenhosas mais freqüentes e abundantes pertenceram ao grupo não-pioneiro e as famílias mais ricas encontradas nas clareiras não diferiram quando comparado à floresta como um todo. Como para as variáveis do estrato herbáceo e da vegetação circundante, os resultados revelaram que efeitos diretos e indiretos da topografia são importantes na determinação da composição interna de clareiras por espécies lenhosas. Estes resultados sugerem que a colonização de clareiras é influenciada não apenas pelas variáveis de estrutura, mas também pela posição topográfica e pelos indivíduos préestabelecidos.

\section{REFERENCES}

Aguiar, O. T. (2003), Comparação entre os métodos de quadrantes e parcelas na caracterização da composição floristica e fitossociológica de um trecho de floresta ombrófila densa no Parque Estadual "Carlos Botelho" - São Miguel Arcanjo, São Paulo. Msc. Dissertation, ESALQ/Universidade de São Paulo, Piracicaba, Brasil.

Barton, A. M.; Fetcher, N. and Redhead, S. (1989), The relationship between treefall gap size and light flux in a Neotropical rain forest in Costa Rica. J. Trop. Ecol., 5, 437-439.

Basnet, K. (1992), Effect of topography on the pattern of trees in Tabonuco (Dacryodes excelsa) dominated rain forest of Puerto Rico. Biotropica, 24, 31-42. 
Bellingham, P. J. and Tanner, E. V. J. (2000), The influence of topography on tree growth, mortality, and recruitment in a tropical montane forest. Biotropica, 32, 378-384.

Brandani, A.; Hartshorn, G. S. and Orians, G. H. (1988), Internal heterogeneity of gaps and species richness in a Costa Rican wet forest. J. Trop. Ecol., 4, 99-119.

Brokaw, N. V. L. (1982), The definition of treefall gap and its effect on measures of forest dynamics. Biotropica, 14, 158-160

Brokaw, N. and Busing, R. T. (2000), Niche versus chance and tree diversity in forest gaps. Trends Ecol. Evol., 15, 183-188.

Brown, N. (1993), The implications of climate and gap microclimate for seedling growth conditions in a Bornean lowland forest. J. Trop. Ecol., 9, 153- 168.

Carvalho, L. M. T.; Fontes, M. A. L. and OliveiraFilho, A. T. (2000), Tree species distribution in canopy gaps and mature forest in an area of cloud forest of the Ibitipoca Range, south-eastern Brazil. Plant Ecol., 149, 9-22.

Chandrashekara, U. M. and Ramakrishnan, P. S. (1994), Vegetation and gap dynamics of a tropical wet evergreen forest in the Western Ghats of Kerala, India. J. Trop. Ecol., 10, 337-354.

Chazdon, R. L. and Fetcher, N. (1984), Photosynthetic light environments in a lowland tropical rain forest in Costa Rica. J. Ecol., 72, 553-564.

Clark, D. B. and Clark, D. A. (1989), The role of physical damage in the seedling mortality regime of a neotropical rain forest. Oikos, 55, 225-230.

Coley, P. D. (1983), Herbivory and defensive characteristics of tree species in a lowland tropical forest. Ecol. Monog., 53, 209-233.

Collins, B. S.; Dunne, K. P. and Pickett, S. T. A. (1985), Responses of forest herbs to canopy gaps. In: Pickett, S. T. A. and White, P. S. (Eds.). The ecology of natural disturbance and patch dynamics. San Diego: Academic Press. pp. 217-234.

Dalling, J. W.; Hubbell, S. P. and Silvera, K. (1998), Seed dispersal, seedling establishment and gap partitioning among tropical pioneer trees. J. Ecol., 86, 674-689.

Denslow, J. S. (1980), Patterns of plant species diversity during succession under disturbance regimes. Oecologia, 46, 18-21.

Denslow, J. S. and Hartshorn, G. S. (1994), Tree-fall gap environments and forest dynamics processes. In: Mcdade, L. A.; Bawa, K. S.; Hespenheide, H. A. and Hartshorn, G. S. (Eds.). La Selva: ecology and natural history of a Neotropical rain forest. Chicago: University of Chicago Press. pp. 120-127.

Domingues, E. N.; Dias, A. C. and Moura Netto, B. V. (1993), O meio biofísico da Bacia do Ribeirão dos Fornos, no Parque Estadual de Carlos Botelho-SP. Rev. Inst. Flor., 5, 203-229.
Gale, N. (2000), The relationship between canopy gaps and topography in a Western Ecuadorian rain forest. Biotropica, 32, 653-661.

Gandolfi, S. (2000), História naturalde uma floresta estacional semidecidual no município de Campinas (São Paulo, Brasil). PhD Thesis, Universidade Estadual de Campinas, Campinas, Brasil.

Garwood, N. C. (1983), Seed germination in a seasonal tropical rain forest in Panama: a community study. Ecol. Monog., 53, 159-181.

George, L. O. and Bazzaz, F. A. (1999), The fern understory as an ecological filter: emergence and establishment of canopy-tree seedlings. Ecology, 80, 833-845.

Hartshorn, G. S. (1978), Tree falls and tropical forest dynamics. In: Tomlinson, P. B. and. Zimmermann, M. H. (Eds.). Tropical trees as living systems. New York: Cambridge University Press. pp. 617-637.

Horvitz, C. C. and Schemske, D. W. (1994), Effects of dispersers, gaps, and predators on dormancy and seedling emergence in a tropical herb. Ecology, 75, 1949-1958.

Hubbell, S. P. and Foster, R. B. (1986), Canopy gaps and the dynamics of a neotropical forest. In: Crawley, M. J. (Ed.). Plant Ecology. Oxford: Blackwell Scientific. pp. 77-96.

Hubbell, S. P.; Foster, R. B.; O’brien, S. T.; Harms, K. E.; Condit, R.; Wechsler, B.; Wright, S. J. and De Lao, S. L. (1999), Light-gap disturbances, recruitment limitation, and tree diversity in a neotropical forest. Science, 283, 554-557.

Jans, L.; Poorter, L.; Van Rompaey, R. S. A. R. and Bongers, F. (1993), Gaps and forest zones in tropical moist forest in Ivory Coast. Biotropica, 25, 258-269.

Klein, R. M. (1980), Ecologia da flora e vegetação do Vale do Itajaí. Sellowia, 32, 165-389.

Lawton, R. O. and Putz, F. E. (1988), Natural disturbance and gap-phase regeneration in a windexposed tropical cloud forest. Ecology, 69, 764-777.

Levey, D. J. (1988), Tropical wet forest treefall gaps and distributions of understory birds and plants. Ecology, 69, 1076-1089.

Lieberman, M.; Lieberman, D. and Peralta, R. (1989), Forests are not just Swiss cheese: canopy stereogeometry of non-gap in tropical forests. Ecology, 70, 550-552.

Lima, R. A. F. (2005), Gap size measurement: The proposal of a new field method. Forest Ecology and Management, 214, 413-419.

Mueller-Dombois, D. and Ellenberg, H. (1974), Aims and methods of vegetation ecology. New York: John Wiley and Sons.

Núñez-Farfán, J. and Dirzo, R. (1988), Within-gap spatial hetereogeneity and seedling performance in a Mexican tropical forest. Oikos, 51, 274-284. 
Oliveira-Filho, A. T.; Curi, N.; Vilela, E. A. and Carvalho, D. A. (1998), Effects of canopy gaps, topography, and soils on the distribution of woody species in a Central Brazilian deciduous forest. Biotropica, 30, 362-375.

Poorter, L.; Jans. L.; Bongers, F. and Van Rompaey, R. S. A. R. (1994), Spatial distribution of gaps along three catenas in the moist forest of Taï National Park, Ivory Coast. J. Trop. Ecol., 10, 385-398.

Raich, J. W. and Christensen, N. L. (1989), Malaysian dipterocarp forest: tree seedling and sapling species composition and small-scale disturbance patterns. Nat. Geog. Res., 5, 348-363.

Robert, A. and Moravie, M. A. (2003), Topographic variation and stand heterogeneity in a wet evergreen forest in India. J. Trop. Ecol., 19, 697-707.

Sanford Jr., R. L.; Braker, H. E. and Hartshorn, G. S. (1986), Canopy opens in a primary neotropical lowland forest. J. Trop. Ecol., 2, 277-282.

Schnitzer, S. A. and Carson, W. P. (2001), Treefall gaps and the maintenance of species diversity in a tropical forest. Ecology, 82, 913-919.

Schupp, E. W. (1988), Seed and early seedling predation in the forest understory and in treefall gaps. Oikos, 51, 71-78.

Tabarelli, M. (1997), A regeneração da Floresta Atlântica Montana. $\mathrm{PhD}$ Thesis, Universidade de São Paulo, São Paulo, Brasil.

Tabarelli, M. and Mantovani, W. (2000), Gap-phase regeneration in a tropical montane forest: the effects of gap structure and bamboo species. Plant Ecol., 148, 149-155.
Uhl, C.; Clark, K.; Dezzeo, N. and Maquirino, P. (1988), Vegetation dynamics in amazon treefall gaps. Ecology, 69, 751-763.

Vázquez-Yanes, C. R. and Orozco-Segovia, A. (1993), Patterns of seed longevity and germination in the tropical rainforest. Ann. Rev. Ecol. Syst., 24, 69-87.

Vázquez-Yanes, C.; Orozco-Segovia, A.; Rincón, E.; Sánchez-Coronado, M. E.; Huante, P.; Toledo, J. R. and Barradas, V. L. (1990), Light beneath the litter in a tropical forest: effect on seed germination. Ecology, 71, 1952-1958.

Whitmore, T. C. (1989), Canopy gaps and the two major groups of forest trees. Ecology, 70, 536-538.

Received: February 10, 2005; Revised: August 30, 2005; Accepted: July 27, 2006. 